\title{
Tratamento Cirúrgico do Câncer Colorretal: Resultados a Longo Prazo e Análise da Qualidade
}

\section{Surgical Treatment of Colorectal Cancer: Long Term Results and Quality Control}

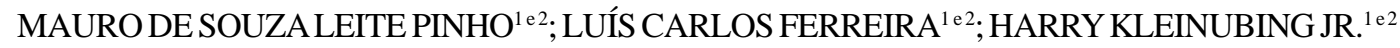 \\ ${ }^{1}$ Cirurgião do Setor de Coloproctologia do Departamento de Cirurgia do Hospital Municipal São José, Joinvile, SC; \\ ${ }^{2}$ Professor da Disciplina de Clínica Cirúrgica do Departamento de Medicina da UNIVILLE - Joinvile, SC - Brasil.
}

PINHO MSL; FERREIRA LC; KLEINUBING Jr. H. Tratamento Cirúrgico do Câncer Colorretal: Resultados a Longo Prazo e Análise da Qualidade. Rev bras Coloproct, 2006;26(4): 422-429.

RESUMO: A obtenção de resultados cada vez melhores no tratamento do câncer colorretal apresenta-se hoje como um desafio devido à sua crescente prevalência em todo o mundo. Diversos estudos têm demonstrado que a qualidade do tratamento cirúrgico instituído representa um dos principais fatores prognósticos, podendo ser esta avaliada através de aspectos como mortalidade operatória, preservação esfincteriana, recorrência local e sobrevida. O objetivo do presente trabalho é apresentar os resultados obtidos a longo prazo no tratamento cirúrgico do câncer colorretal pelo Grupo de Coloproctologia do Departamento de Cirurgia do Hospital Municipal São José, em Joinville, e confrontá-los com a literatura a respeito, visando obter uma avaliação crítica da qualidade do tratamento instituído. Foi realizada uma análise prospectiva de uma série consecutiva de 97 pacientes submetidos ao tratamento cirúrgico do câncer colorretal, com perspectivas curativas e tempo de seguimento médio de 80,8 meses. Foi observada mortalidade operatória em seis pacientes $(6,1 \%)$, recidiva local para câncer retal e colônico em seis $(\mathbf{1 2 , 5 \% )}$ e quatro $(\mathbf{9 , 7 \%})$ pacientes, respectivamente, e a necessidade de realização de colostomia definitiva no câncer retal em 14 casos (27\%). A sobrevida geral média foi de 48,9 meses. A sobrevida geral de cinco anos para o seguimento oncológico $(n=63)$ foi de $52 \%$, sendo $89 \%$ para pacientes estágio 1, $70 \%$ para pacientes estágio 2 e $20 \%$ para pacientes estágio 3. Concluímos que o tratamento instituído encontra-se dentro dos padrões aceitáveis do ponto de vista da literatura, demonstrando, no entanto, a necessidade de aprimoramento em alguns aspectos específicos.

Descritores: Neoplasias colorretais, cirurgia, controle de qualidade.

A obtenção de resultados cada vez melhores no tratamento cirúrgico do câncer colorretal apresenta-se hoje como um desafio permanente, devido à sua crescente prevalência em todo o mundo. Embora tenha ocorrido uma importante redução da morbidade e mortalidade operatórias ao longo das últimas décadas em conseqüência de melhores cuidados pré e pós operatórios, os resultados oncológicos a longo prazo não apresentaram uma variação semelhante, persistindo os níveis de sobrevida bastante próximos àqueles observados no início do século passado. Visando melhorar estes resultados, sucessivas alterações têm sido propostas em diferentes aspectos do tratamento ${ }^{1}$.

No que diz respeito à técnica operatória, tentativas de ampliação da radicalidade através de ligadu- ras vasculares altas ou linfadenectomias pélvicas alargadas mostraram-se controversas quanto à sua efetividade ${ }^{2}$, enquanto a ressecção do mesorreto intacto representou um real avanço no sentido de reduzir a incidência de recidivas locais ${ }^{3}$.

Outros importantes benefícios em relação à obtenção de melhores resultados oncológicos tem sido obtidos através do aprimoramento de terapias adjuvantes incluindo a quimioterapia e radioterapia ${ }^{4}$. Inicialmente pouco eficazes, estas terapias representam hoje um papel fundamental e indispensável no tratamento do câncer colorretal, em especial após a instituição de doses maiores de radioterapia e pela compreensão de seu efeito sinérgico com a quimioterapia. A introdução da terapia neo-adjuvante representou ou-

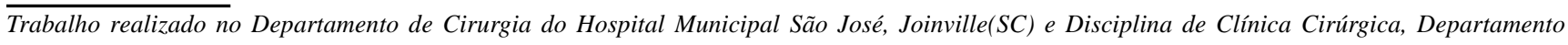
de Medicina, UNIVILLE, Joinville (SC).

$\overline{\text { Recebido em 14/09/2006 }}$

Aceito para publicação em 04/10/2006 
tro marco no tratamento do câncer retal, oferecendo a possibilidade de uma regressão tumoral parcial ou total, vindo a se constituir como um elemento terapêutico capaz de reduzir índices de recidiva local e sobrevida. O desenvolvimento de técnicas de biologia molecular representa hoje ainda uma importante perspectiva através de uma melhor caracterização da neoplasia e o desenvolvimento de medicamentos específicos, em especial através de terapias monoclonais ${ }^{5}$.

Entretanto, talvez a maior ênfase atual a respeito dos fatores prognósticos relevantes no tratamento do câncer colorretal esteja relacionada à qualidade do tratamento cirúrgico instituído. Diversos estudos têm demonstrado um benefício significativo em relação a aspectos como mortalidade operatória, preservação esfincteriana, recorrência local e sobrevida em centros nos quais cirurgiões especialistas em cirurgia colorretal realizam um volume maior de casos do que aquele observado em centros não especializados e com um menor volume ${ }^{6-8}$.

Neste sentido, torna-se de grande relevância o conhecimento dos resultados obtidos a curto e longo prazo nos diferentes centros que realizam o tratamento do câncer colorretal, visando uma melhor definição dos critérios de qualidade a partir de estudos comparativos com aqueles obtidos em outros centros.

O objetivo do presente trabalho é apresentar os resultados obtidos a longo prazo no tratamento cirúrgico do câncer colorretal pelo Grupo de Coloproctologia do Departamento de Cirurgia do Hospital Municipal São José, em Joinville, Santa Catarina, assim como confrontá-los com a literatura a respeito, visando obter uma avaliação crítica da qualidade do tratamento instituído, assim como detectar a necessidade de eventuais revisões de conduta.

\section{MÉTODOS}

Foi feita uma análise prospectiva de uma série consecutiva de pacientes submetidos ao tratamento cirúrgico do câncer colorretal no Departamento de Cirurgia do Hospital Municipal São José com perspectivas curativas e com tempo de seguimento mínimo de 24 meses. 97 pacientes preencheram estes critérios e foram incluídos no estudo atingindo um tempo de seguimento médio de 80,8 meses ou 6,7 anos. Todos os pacientes foram operados pelos dois primeiros autores (MP 86 - LCF 11) ou por residentes sob sua supervisão direta no campo operatório.

Todos os casos foram inseridos desde o início da casuística em uma planilha contendo os dados mais relevantes, sendo os pacientes seguidos ambulatorialmente através de revisões periódicas com intervalos não superiores a seis meses, incluindo no mínimo um exame físico, dosagem de CEA sanguíneo, radiografias simples de tórax e ultrassonografia abdominal. Pacientes faltantes ao seguimento foram rastreados através de contatos telefônicos e solicitados a retornar para avaliação. Duas pacientes foram perdidas do controle e foram excluídas das análises de sobrevida, sendo conseqüentemente possível um seguimento até a presente data de 95 pacientes ou $98 \%$ da casuística.

O tratamento cirúrgico instituído em todo o grupo obedeceu ao padrão convencional de ressecções, seguindo os princípios oncológicos que incluem a linfadenectomia e, no caso dos tumores retais, a ressecção do mesorreto intacto. Não foram realizadas ressecções por via laparoscópica na presente casuística.

A terapia adjuvante foi indicada para a maior parte dos tumores colônicos estágio $2 \mathrm{~b}$ ou superior e a terapia neoadjuvante foi utilizada em pacientes portadores de tumores retais fixos ou volumosos.

A análise da curva de sobrevida foi realizada através da utilização do programa SSPS®.

\section{RESULTADOS}

Observou-se neste grupo um discreto predomínio do sexo masculino ( $\mathrm{n}=51)$ sobre o sexo feminino $(\mathrm{n}=46)$ e a média de idade foi de 61 anos $(28-87)$. A localização mais freqüente foi no reto $(52,5 \%)$, conforme demonstrado na tabela 1 .

As operações realizadas estão demonstradas na tabela 2, havendo um amplo predomínio das retosigmoidectomias abdominais $(45,3 \%)$. Nos pacientes portadores de câncer retal $(n=51)$ houve a necessidade de realização de retosigmoidectomia abdominoperineal em 14 casos (27\%) devido ao comprometimento do canal anal ou ausência de margem de segurança oncológica.

Tabela 1 - Localização dos tumores colorretais.

\begin{tabular}{lrr}
\hline Local & $\mathbf{n}$ & $\mathbf{\%}$ \\
\hline Reto & 51 & $52,5 \%$ \\
Colon sigmóide & 21 & $21,6 \%$ \\
Colon direito & 13 & $13,4 \%$ \\
Colon esquerdo & 6 & $6,1 \%$ \\
Colon transverso & 6 & $6,1 \%$ \\
\hline
\end{tabular}


Tabela 2 - Tipos de ressecções realizadas.

\begin{tabular}{lrc}
\hline Retossigmoidectomia abdominal & 44 & $45,3 \%$ \\
Retossigmoidectomia abdominoperineal & 14 & $14,4 \%$ \\
Colectomia direita & 14 & $14,4 \%$ \\
Sigmoidectomia & 12 & $12,3 \%$ \\
Colectomia esquerda & 6 & $6,1 \%$ \\
Colectomia de transverso & 4 & $4,1 \%$ \\
Colectomia total & 1 & $1 \%$ \\
Proctocolectomia total & 1 & $1 \%$ \\
Excisão local & 1 & $1 \%$ \\
\hline
\end{tabular}

Tabela 3 - Sobrevida de 5 anos.

\begin{tabular}{lrc}
\hline Autor & n & Sobrevida 5 a. \\
\hline Kerr e cols, 2005 & 936 & $62,2 \%$ \\
Hilska e cols, 2004 & 387 & $57 \%$ \\
Singh e cols, 1989 & 640 & $67 \%$ \\
Rabeneck e cols, 2004 & 22633 & $52,1 \% *$ \\
& & $48,3 \% * *$ \\
Esta série & 63 & $52 \%$ \\
\hline
\end{tabular}

* Hospitais com grande volume.

** Hospitais de baixo volume.

O estadiamento pela classificação TNM demonstrou a presença de tumores estágio 1 em 21 pacientes (21,6\%), estágio 2 em 40 (41,2\%), estágio 3 em 33 (34\%) e em três pacientes não foi possível estadiar adequadamente a partir do laudo anatomopatológico.

\section{Mortalidade operatória}

Na presente série ocorreram seis óbitos $(6,1 \%)$ no período pós-operatório, sendo três destes em de- corrência de peritonite e os outros três por problemas de natureza clínica (embolia pulmonar, pneumonia e insuficiência cardíaca). A média de idade entre estes pacientes foi de 75,5 anos $(63-87)$.

\section{Recidiva}

Dentre os 89 pacientes avaliados a longo prazo o seguimento médio foi de 81 meses $(24-142)$. Destes, 56 pacientes não apresentaram sinais de recidiva $(62,9 \%)$, enquanto em 33 pacientes ocorreu recorrência da doença $(37,1 \%)$. Ao analisarmos de acordo com o estadiamento, observamos para os estágios 1,2 e 3 os índices de recidiva de $10 \%, 25 \%$ e $64,5 \%$, respectivamente.

A recidiva local foi observada em seis pacientes submetidos ao tratamento do câncer retal $(12,5 \%)$ e em quatro pacientes portadores de câncer colônico $(9,7 \%)$.

\section{Sobrevida geral}

No presente momento, $51(53,6 \%)$ pacientes permanecem vivos, sendo que quatro destes encontram-se com recidiva da doença.

A sobrevida média em todos os 89 pacientes avaliados a longo prazo foi de 48,6 meses. Nos pacientes portadores de tumores estágio 1 , a sobrevida média foi de 65 meses, sendo que um paciente faleceu de outra causa, sem evidência de recidiva. Nos casos estágio 2 e 3 esta sobrevida foi de 57,7 e 34,8 meses, respectivamente, sendo que cinco pacientes estágio 2 e três pacientes estágio 3 faleceram de outras causas. As curvas de sobrevida total e de acordo com o estágio estão demonstradas na figura 1 .

\section{Figura 1 - Curvas de sobrevida.}

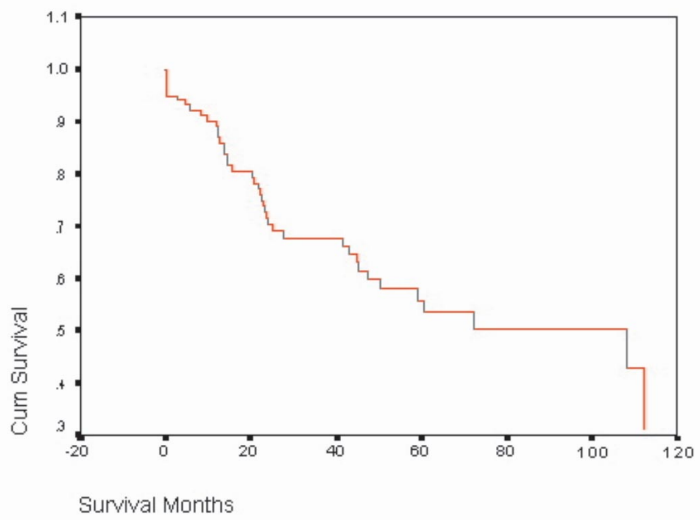

Geral

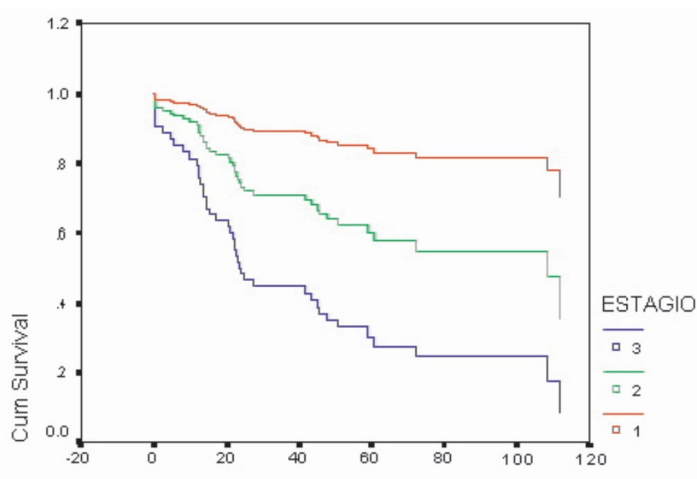

Survival Months

Por estágios 


\section{Sobrevida de cinco anos}

Ao analisarmos os 63 pacientes desta série com seguimento mínimo de 60 meses ou cinco anos, veremos que $37(58,7 \%)$ já faleceram, sendo seis destes no pós-operatório imediato e sete, de causas não relacionadas à doença ou ao tratamento cirúrgico. Vinte e seis pacientes $(41,2 \%)$ permanecem vivos, nenhum deles com sinais de recidiva da doença, sendo, portanto esta a sobrevida geral, não corrigida, de cinco anos. Ao corrigirmos este cálculo de sobrevida através da exclusão dos óbitos pós-operatórios e mortes por causas não relacionadas à doença a fim de obtermos uma avaliação dos resultados oncológicos a longo prazo observaremos uma sobrevida geral de cinco anos de $52 \%$, sendo $89 \%$ para pacientes estágio $1,70 \%$ para pacientes estágio 2 e $20 \%$ para pacientes estágio 3.

\section{DISCUSSÃO}

Diversos estudos têm sido realizados com o objetivo de definir o impacto do cirurgião como fator prognóstico no tratamento do câncer colorretal ${ }^{6-31}$. Para atingir este objetivo, tem-se buscado analisar seu potencial qualitativo para a realização deste tratamento e confrontá-lo com os resultados efetivamente obtidos.

A qualidade do tratamento tem sido avaliada através de alguns parâmetros potencialmente relacionados ao tratamento cirúrgico, incluindo a mortalidade operatória, ocorrência de deiscências anastomóticas, número de colostomias definitivas, recidivas tumorais no local da ressecção e índices de sobrevida de cinco $\operatorname{anos} 14,18,21,23$.

O potencial qualitativo do cirurgião para o tratamento do câncer colorretal tem sido avaliado através de dois aspectos inter-relacionados, sendo estes a especificidade de sua formação e a regularidade de sua experiência, usualmente quantificada a partir do número de ressecções colorretais anuais ${ }^{8,13-15}$.

Para avaliar o valor prognóstico da formação do cirurgião, diversos estudos têm sido realizados comparando-se os resultados obtidos por cirurgiões colorretais especializados com aqueles operados por cirurgiões em fase de formação ou não-especialistas.

Smith e cols ${ }^{14}$ realizaram uma auditoria prospectiva em 5.173 pacientes portadores de câncer colorretal e observaram resultados significativamente melhores obtidos por cirurgiões especialistas, quando comparados ao tratamento realizado por não especialistas referentes à mortalidade operatória (OR 0.67), deiscências anastomóticas (OR 0.46), recorrência local (OR 0.44) e sobrevida (OR 0.76).

Read e cols ${ }^{15}$ publicaram uma revisão retrospectiva realizada por médicos radioterapêutas, incluindo 384 pacientes submetidos ao tratamento cirúrgico do câncer retal após radioterapia neoadjuvante, com o objetivo de comparar resultados obtidos por cirurgiões colorretais $(n=251)$ e não colorretais $(n=133)$. Os pacientes operados por especialistas apresentaram melhores resultados em relação à sobrevida de cinco anos $(77 \%$ x $68 \%, \mathrm{p}<0,005)$ e ao controle local da doença $(93 \% \times 84 \%, \mathrm{p}<0,005)$.

Resultados semelhantes foram observados por Meagher ${ }^{6}$ em uma revisão sistemática no MEDLINE em um período de nove anos, concluindo o autor que as informações atuais sugerem fortemente que o cirurgião realmente representa um importante fator prognóstico no tratamento do câncer colorretal.

Quanto aos resultados obtidos por cirurgiões em treinamento sob supervisão direta de especialistas, estudos realizados por Renwick e cols ${ }^{10} \mathrm{e}$ Chester e cols ${ }^{13}$ não foram capazes de demonstrar diferenças significativas em relação àqueles resultantes do tratamento realizado pelos próprios especialistas.

A série apresentada neste estudo representa a casuística do Departamento de Cirurgia de um hospital público, classificado como universitário pelo Ministério da Saúde e que inclui atividades de ensino a nível de Graduação e Residência Médica. O Grupo de Coloproctologia realiza atualmente uma média aproximada de 70 operações colorretais abdominais por ano, das quais cerca de metade refere-se a ressecções relacionadas ao câncer colorretal.

Embora essencial para a avaliação dos resultados do tratamento adequado de neoplasias malignas, o seguimento a longo prazo destes pacientes representa um grande desafio, requerendo o estabelecimento de rotinas rígidas e permanente reavaliação dos procedimentos. Três aspectos nos parecem particularmente importantes para atingir este objetivo. $\mathrm{O}$ primeiro deles é a existência de um único banco de dados, independentemente do cirurgião responsável pelo caso. O segundo refere-se a um ambulatório de fácil acesso aos pacientes deste banco de dados, em 
horário fixo e não submetido a mudanças freqüentes, o qual deverá ser realizado, se possível, pelo mesmo cirurgião responsável pela inserção e manutenção do banco de dados. Finalmente, deve-se dar uma extrema atenção em cada consulta à atualização de informações capazes de permitir a localização de pacientes eventualmente faltantes, como telefones, endereços, parentes ou outras pessoas de referência. Devese destacar que o grande crescimento do acesso por parte da população de baixa renda à telefonia fixa ou celular ao longo da última década representou uma importante contribuição para o seguimento de pacientes em nosso país.

O estabelecimento desta rotina permitiu alcançarmos um seguimento de $98 \%$ dos pacientes com uma duração média de 6,7 anos. Para preservar a integridade de nosso protocolo, incluímos neste seguimento apenas pacientes operados por cirurgiões do Grupo de Coloproctologia, não sendo considerados pacientes operados por outros cirurgiões do Departamento, incluindo operações eletivas ou de urgência.

A análise destes dados forneceu algumas informações bastante relevantes para uma avaliação qualitativa do tratamento instituído, em particular através da comparação com a literatura, como discutiremos a seguir:

\section{Proporção de ressecções abdominoperineais}

Meyerhardt e cols ${ }^{29}$ observaram, a partir de um estudo multicêntrico com 1330 pacientes, que a proporção de ressecções abdominoperineais e a conseqüente realização de colostomias definitivas no tratamento do câncer retal está relacionada ao volume de casos operados em cada serviço, sendo estas realizadas em 46,3\% naqueles de baixo volume, $41,3 \%$ nos serviços de volume intermediário e $31,8 \%$ em serviços de alto volume. Penninckx ${ }^{19}$, em uma revisão da literatura, afirma que a incidência de ressecções abdominoperineais em portadores de câncer retal varia entre $23-58 \%$ em centros especializados e $43-57 \%$ em serviços de cirurgia geral e sugere que níveis abaixo de $40 \%$ sejam desejáveis para a ocorrência deste procedimento.

$\mathrm{Na}$ presente série, apesar de esforços bastante determinados em ampliar ao máximo o número de operações com preservação esfincteriana, otimizando a dissecção retal até o nível do canal anal e empregando em alguns casos recursos téc- nicos alternativos como a anastomose por via transanal, eversão do reto ou rebaixamento endoanal, não foi possível evitar a colostomia definitiva em $27 \%$ dos tumores definidos como retais, demonstrando a elevada incidência de neoplasias que comprometem parte do canal anal ou com grande proximidade a este.

\section{Mortalidade operatória}

Dimick e cols ${ }^{18}$ realizaram um importante estudo analisando os registros de 20862 pacientes submetidos ao tratamento cirúrgico do câncer colorretal nos Estados Unidos no ano de 1997, com ênfase especial sobre a mortalidade operatória, idade do paciente e volume de casos operados em cada hospital. A mortalidade operatória geral para os 842 hospitais foi de $3,1 \%$. A análise por faixa etária revelou uma variação dos níveis de mortalidade que oscilou de $0,8 \%$ para pacientes com idade abaixo de 50 anos até $6,9 \%$ para aqueles com mais de 80 anos. Hospitais com grande volume de casos operados (>150 casos/ano) apresentaram uma mortalidade operatória significativamente menor do que aqueles com menor volume ( $<55$ casos/ano) $(2,5 \%$ x 3,7\%, $\mathrm{p}=0,006)$. Esta diferença foi resultante dos resultados obtidos em pacientes mais idosos, observandose uma mortalidade maior nos hospitais de menor volume para pacientes acima de 65 anos $(4,5 \% \mathrm{x}$ $3,1 \%, \mathrm{p}=0,03)$ e acima de 80 anos $(7,3 \%$ x $4,6 \%$, $\mathrm{p}=0,04)$.

Esta relação entre a idade e a mortalidade operatória mostrou-se também bastante relevante na série aqui apresentada. Embora a mortalidade operatória geral de $6,1 \%$ entre os 97 pacientes esteja em um nível discretamente acima da faixa de 2-5\% presente na maior parte das estatísticas, ela é o resultado de uma grande discrepância entre a mortalidade operatória obtida nas diferentes faixas etárias, tendo esta sido de $0 \%$ em pacientes até 50 anos $(n=24), 4,9 \%$ para os pacientes entre 51 e 75 anos $(n=61)$ e $25 \%$ para aqueles com idade superior a 75 anos $(n=12)$. A elevada mortalidade operatória neste último grupo revela a necessidade de revisão dos cuidados pré, intra e pós-operatórios destes pacientes uma vez que deverão estar mais relacionados à ocorrência de complicações do que à técnica operatória propriamente dita, realizada de forma padronizada em pacientes de todas as faixas etárias. 


\section{Recidivas locais}

A incidência de recidivas locais é considerada como um dos principais aspectos potencialmente capazes de revelar a acuidade da técnica operatória empregada. No que diz respeito às ressecções de tumores colônicos, os relatos sobre a recorrência locorregional é bastante difícil de ser analisada devido à grande variabilidade entre os diferentes relatos, os quais apresentam números que podem variar entre $6 \%$ e $28 \%$. Neste sentido, a ocorrência de recidiva locorregional em $9,7 \%$ dos tumores colônicos incluídos nesta série parecem situar-se dentro de uma faixa compatível com os resultados esperados.

Ao contrário dos tumores do cólon, a recidiva local no tratamento cirúrgico dos tumores do reto tem sido tema de um grande número de estudos ao longo dos últimos anos, em particular pela introdução e desenvolvimento de novos conceitos como a ressecção total do mesorreto e a utilização de terapia neo-adjuvante com resultados cada vez mais efetivos. Com a utilização destes recursos de forma adequada, observou-se uma progressiva redução da incidência de recidiva local nos relatos da literatura, sendo hoje considerada como aceitável a obtenção de níveis situados entre 5\% e $10 \%$.

Em nossa casuística, a incidência de 12,5\% de recidivas locais, embora bastante próxima aos níveis considerados aceitáveis, pode ter sido influenciada pela utilização de bomba de cobalto como equipamento para a realização de radioterapia, uma vez que o número de respostas completas mostrou-se bastante inferior àqueles relatados pela literatura em geral com a utilização dos modernos aparelhos aceleradores lineares. Esta questão deverá ser resolvida em breve através da instalação de um equipamento destes em nosso Serviço de Oncologia. Além disto, novos esforços técnicos deverão ser cumpridos no sentido de obter-se uma maior redução da recidiva local, incluindo relatos recentes que sugerem a realização de uma dissecção mais alargada no terço inferior do reto, como aquele publicado por Nagtegaal e cols ${ }^{32}$.

\section{Sobrevida}

Ao observarmos as curvas de sobrevida na presente série, observamos uma estreita correlação com o estadiamento da doença, apresentando em sua maioria níveis compatíveis com aqueles relatados na literatura em geral. A análise corrigida do grupo com segui- mento superior a cinco anos demonstrou uma sobrevida geral de cinco anos de $52 \%$.

A análise comparativa deste resultado com a literatura representa um grande desafio devido à ampla variabilidade entre os diferentes autores. De uma forma geral, análises de sobrevida em centros especializados com grande volume tendem a demonstrar níveis mais elevados quando comparados a hospitais e grupos com menor volume e/ou nível de especialização. Neste sentido, nossa sobrevida de cinco anos mostrou-se dentro da faixa média aceitável na maior parte da literatura, embora em seu limiar inferior, uma vez que esta irá variar entre 50 e $65 \%$, conforme observado na Tabela 3. Uma análise mais detalhada desta sobrevida geral nos revela que ela foi negativamente influenciada por um resultado insatisfatório a longo prazo especificamente observado nos pacientes estágio 3, cuja sobrevida de $20 \%$ foi bastante inferior ao esperado, usualmente relatado na faixa de 30\%-40\%. Em relação aos pacientes portadores de tumores estágio 1 e 2, obtivemos uma sobrevida de cinco anos de $89 \%$ e $70 \%$, sendo estes compatíveis com a maior parte dos relatos da literatura.

Assim sendo, concluímos que a presente revisão da experiência a longo prazo no tratamento do câncer colorretal em nosso Departamento logrou seu objetivo de possibilitar uma avaliação de sua qualidade. De uma forma geral, obtivemos como resposta que o tratamento instituído encontra-se dentro dos padrões aceitáveis do ponto de vista da literatura, demonstrando ainda, no entanto, alguns aspectos específicos sobre os quais necessitamos dedicar alguma atenção no sentido de obter um aprimoramento dos resultados.

Além disto, gostaríamos de destacar a validade do esforço desempenhado no sentido de manter um rigoroso seguimento a longo prazo dos pacientes, o qual possibilitou o conhecimento de nossos resultados, como base para o constante aprimoramento, estratégia obrigatória não apenas para serviços de grande volume, mas pertinente a todo cirurgião dedicado ao tratamento do câncer colorretal, independentemente de seu volume pessoal e institucional.

\section{AGRADECIMENTO}

Os autores agradecem ao Dr. Cristian Garcia pela colaboração na elaboração das curvas de sobrevida. 
ABSTRACT: Improvement of results in treatment of colorectal cancer remains a major challenge due to its increasing worldwide prevalence. Several studies have demonstrated the quality of surgical treatment as one of the most important prognostic factors, assessed by parameters such as operative mortality, sphincter preservation, local recurrence and survival rates. The aim of this study is to present the long term results of Coloproctology Group of Department of Surgery of Hospital Municipal São José, in Joinville, Brazil, as well as its quality analysis according to the accepted standards from literature. A prospective analysis of a consecutive series of 97 colorectal cancer patients operated for curative purpose was undertaken, with a mean follow up of 80,8 months. Operative mortality occurred in six patients $(6,1 \%)$, local recurrence for recatl and colon cancer in six $(12,5 \%)$ and four $(9,7 \%)$ patients, respectively, and abdominoperineal resection was performed in 14 cases $(27 \%)$ for rectal cancer. Mean overall survival was 48,9 months. Five-year overall oncological survival (n=63) was $52 \%$, including $89 \%$ for stage1, $70 \%$ for stage 2 and $20 \%$ for stage 3 patients. We conclude that the results obtained are acceptable for literature standards, but some specific parameters need to be improved.

Key words: Colorectal neoplasms, surgery, quality control.

\section{REFERÊNCIAS}

1. Rossi BM, Nakagawa WT, Ferreira FO, Aguiar Jr. S, Lopes A. Câncer de colon reto e ânus.São Paulo: Lemar e TecMedd Editora, 2004.

2. Moreira LF, Hizuta A, , Tanaka N, Orita K. Lateral lymph node dissection for rectal carcinoma below the peritoneal reflection. Br J Surg. 1994 Feb;81(2):293-6.

3. Birbeck KF, Macklin CP, Tiffin NJ, Parsons W, Dixon MF, Mapstone NP et al. Rates of circumferential resection margin involvement vary between surgeons and predict outcomes in rectal cancer surgery. Ann Surg. 2002 Apr;235(4):449-57

4. Ferrigno R e David Filho WJ. Radioterapia e quimioterapia pré-operatórias em câncer de reto. In: Rossi BM, Nakagawa WT, Ferreira FO, Aguiar Jr. S, Lopes A. Câncer de colon, reto e ânus.São Paulo: Lemar e TecMedd Editora, 2004. P.263273.

5. Pinho M. Anticorpos monoclonais no tratamento do câncer colorretal: fundamentos e estado atual. Rev Bras Coloproctologia 2005:24; 382-4.

6. Meagher AP. Colorectal cancer: is the surgeon a prognostic factor? A systematic review. Med J Aust. 1999 Sep 20;171(6):308-10.

7. Dorrance HR, Docherty GM, O'Dwyer PJ. Effect of surgeon specialty interest on patient outcome after potentially curative colorectal cancer surgery. Dis Colon Rectum. 2000 Apr;43(4):492-8.

8. Hilska M, Roberts PJ, Kossi J, Paajanen H, Collan Y, Laato $M$. The influence of training level and surgical experience on survival in colorectal cancer. Langenbecks Arch Surg. 2004 Nov;389(6):524-31.

9. Singh KK, Barry MK, Ralston P, Henderson MA, McCormick JS, Walls AD et al. Audit of colorectal cancer surgery by nonspecialist surgeons. Br J Surg. 1997 Mar;84(3):343-7.

10. Renwick AA, Bokey EL, Chapuis PH, Zelas P, Stewart PJ, Rickard MJ, Dent OF. Effect of supervised surgical training on outcomes after resection of colorectal cancer. Br J Surg. 2005;92(5):631-6.
11. Ubhi SS, Kent SJ. Which surgeons in a district general hospital should treat patients with carcinoma of the rectum? J R Coll Surg Edinb. 1995;40(1):52-4.

12. Hermanek P, Mansmann U, Staimmer DS, Riedl S, Hermanek $P$. The German experience: the surgeon as a prognostic factor in colon and rectal cancer surgery. Surg Oncol Clin N Am. 2000;9(1):33-49, vi.

13. Chester J, Britton D. Elective and emergency surgery for colorectal cancer in a district general hospital: impact of surgical training on patient survival. Ann R Coll Surg Engl. 1989;71(6):370-4.

14. Smith JA, King PM, Lane RH, Thompson MR. Evidence of the effect of 'specialization' on the management, surgical outcome and survival from colorectal cancer in Wessex. Br J Surg. 2003;90(5):583-92.

15. Read TE, Myerson RJ, Fleshman JW, Fry RD, Birnbaum EH, Walz BJ, Kodner IJ. Surgeon specialty is associated with outcome in rectal cancer treatment. Dis Colon Rectum. 2002 ;45(7):904-14.

16. Martling A, Cedermark B, Johansson H, Rutqvist LE, Holm T. The surgeon as a prognostic factor after the introduction of total mesorectal excision in the treatment of rectal cancer. Br J Surg. 2002;89(8):1008-13.

17. Solomon MJ, Thomas RJ, Gattellari M, Ward JE. Does type of surgeon matter in rectal cancer surgery? Evidence, guideline consensus and surgeons' views. ANZ J Surg. $2001 ; 71(12): 711-4$.

18. Dimick JB, Cowan JA Jr, Upchurch GR Jr, Colletti LM. Hospital volume and surgical outcomes for elderly patients with colorectal cancer in the United States. J Surg Res. 2003;114(1):50-6.

19. Penninckx F. Surgeon-related aspects of the treatment and outcome after radical resection for rectal cancer. Acta Gastroenterol Belg. 2001;64(3):258-62.

20. Renzulli P, Laffer UT. Learning curve: the surgeon as a prognostic factor in colorectal cancer surgery. Recent Results Cancer Res. 2005; 165:86-104.

21. Schrag D, Panageas KS, Riedel E, Hsieh L, Bach PB, Guillem JG, Begg CB. Surgeon volume compared to hospital volume 
as a predictor of outcome following primary colon cancer resection. J Surg Oncol. 2003;83(2):68-78; discussion 78-9.

22. Paimela $\mathrm{H}$, Lindstrom $\mathrm{O}$, Tomminen $\mathrm{T}$, Iivonen $\mathrm{M}$, Kononen E, Kuusanmaki P. Surgery for colorectal cancer in a low-volume unit: assessment of key issues in the achievement of acceptable clinical results. Int $\mathbf{J}$ Gastrointest Cancer. 2005;35(3):205-10.

23. Rabeneck L, Davila JA, Thompson M, El-Serag HB. Surgical volume and long-term survival following surgery for colorectal cancer in the Veterans Affairs Health-Care System. Am J Gastroenterol. 2004 Apr;99(4):668-75.

24. McGrath DR, Leong DC, Gibberd R, Armstrong B, Spigelman AD. Surgeon and hospital volume and the management of colorectal cancer patients in Australia. ANZ J Surg. 2005;75(10):901-10.

25. Peeters KC, van de Velde CJ. Surgical quality assurance in rectal cancer treatment: the key to improved outcome. Eur J Surg Oncol. 2005;31(6):630-5.

26. Engel J, Kerr J, Eckel R, Gunther B, Heiss M, Heitland W et al. Influence of hospital volume on local recurrence and survival in a population sample of rectal cancer patients. Eur J Surg Oncol. 2005;31(5):512-20.

27. Kerr J, Engel J, Eckel R, Holzel D. Survival for rectal cancer patients and international comparisons. Ann Oncol. 2005;16(4):664-72.
28. McArdle CS, Hole DJ. Influence of volume and specialization on survival following surgery for colorectal cancer. Br J Surg. 2004;91(5):610-7.

29. Meyerhardt JA, Tepper JE, Niedzwiecki D, Hollis DR, Schrag $\mathrm{D}$, Ayanian JZ et al. Impact of hospital procedure volume on surgical operation and long-term outcomes in high-risk curatively resected rectal cancer: findings from the Intergroup 0114 Study. J Clin Oncol. 2004 Jan 1;22(1):166-74.

30. Urbach DR, Bell CM, Austin PC. Differences in operative mortality between high- and low-volume hospitals in Ontario for 5 major surgical procedures: estimating the number of lives potentially saved through regionalization. CMAJ. 2003;168(11):1409-14.

31. Schrag D, Panageas KS, Riedel E, Cramer LD, Guillem JG, Bach PB, Begg CB. Hospital and surgeon procedure volume as predictors of outcome following rectal cancer resection. Ann Surg. 2002;236(5):583-92.

32. Nagtegaal ID, van de Velde CJ, Marijnen CA, van Krieken JH, Quirke P. Low rectal cancer: a call for a change of approach in abdominoperineal resection. J Clin Oncol. 2005 Dec 20;23(36):9257-64.

\section{Endereço para Correspondência:}

MAURO PINHO

Rua Palmares 380 - Atiradores

89.203-230 - Joinville (SC)

mauro.pinho@terra.com.br 